\title{
Joint optimization of BS-VS association and power control in secure HSR communication systems
}

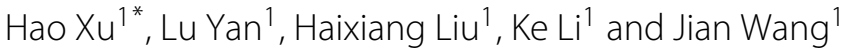

\author{
*Correspondence: \\ 18612113434@163.com \\ 'China Academy of Railway \\ Sciences, Beijing, China
}

\begin{abstract}
In this paper, the secure transmission for high-speed railway (HSR) communication system is studied. The considered HSR wireless communication system consists of a macro base station, B roadside base stations, and K vehicle stations (VSs) on the top of the train, and the eavesdropping user is a mobile unmanned aerial vehicle (UAV). We consider maximizing the sum of the minimum security rate of each time slot as the objective function, and the constraint conditions contain the quality of service (QoS), switch requirements and the total power constraint. The original optimization problem is mixed-integer and non-convex, it is intractable to solve directly. The block coordinate descent (BCD) method is applied, and the original problem can be decoupled into two sub-problems. The one is the joint BS-VS association problem, and the other is the power control problem. The first sub-problem of optimizing BS-VS association can be solved by the successive convex approximation (SCA) algorithm, and the second one of optimizing transmit power can be solved by the lagrangian dual method. Simulation results show that the proposed algorithms have good convergence.
\end{abstract}

Keywords: Secure transmission, HSR communication system, Eavesdropping user, $U A V$, Security rate

\section{Introduction}

As a kind of comfortable, fast, and safe traffic tool, the application of high-speed railway (HSR) has attracted the extensive attention in many countries. Especially in China, the HSR has developed rapidly and has become the main tool of medium and long-distance passenger transport. The widespread popularity of HSR poses a severe challenge to the HSR communication service of train operation and passenger communication service $[1,2]$. In order to ensure the safety of train operation, the train multimedia dispatching, railway emergency communication and HSR line condition monitoring based the broadband railway communication system are becoming more and more important [3]. In addition, it is necessary to provide high-quality voice services, video services, and Internet access for passengers on the train [4]. In order to satisfy the requirements of the data transmission rate in HSR communication, the broadband mobile communication system

(C) The Author(s). 2021 Open Access This article is licensed under a Creative Commons Attribution 4.0 International License which permits use, sharing, adaptation, distribution and reproduction in any medium or format, as long as you give appropriate credit to the original author(s) and the source, provide a link to the Creative Commons licence, and indicate if changes were made. The images or other third party material in this article are included in the article's Creative Commons licence, unless indicated otherwise in a credit line to the material. If material is not included in the article's Creative Commons licence and your intended use is not permitted by statutory regulation or exceeds the permitted use, you will need to obtain permission directly from the copyright holder. To view a copy of this licence, visit http://creativecommons.org/licenses/by/4.0/. 
for HSR has attracted wide attention in industry and academia [5]. So as to better improve the QoS, LTE for railway (LTE-R) is proposed by the International Union of Railways to substitute for the global system for mobile communications for railway (GSM-R) [6]. The fifth generation (5G) communication system will cover a variety of high mobility scenarios, such as HSR, highway, and subway. Due to the large bandwidth, 5G communication system for railway (5G-R) can provide high-speed data service for the train operation and passengers at the same time. It is predicted that the peak data rate of HSR communication in the future will reach $0.5-5$ Gbps [7].

The most promising solution is to adopt the two hop architecture in the future HSR communication system [8-10]. The first hop from the base station (BS) to the mobile relay station (MRS) on the top of the train works in a lower frequency band, which can avoid the penetration loss of the train and reduce the probability of handover failure $[11,12]$. The other hop is from MRS to the mobile station (MS) in the train, which can use the millimeter wave to obtain the high bandwidth [13]. A HSR wireless communications between a fixed BS and a high speed train (HST) was considered, and a beam-forming method with Doppler suppression for the BS was proposed, which could maximize the signal-to-interference-plus-noise ratio [14]. The weight was computed from the direction of departure of two direct paths, a position aided distributed beamforming algorithm was proposed in [15]. The joint optimization of access control and resource allocation for multimedia services in HSR wireless networks was studied in [16]. By introducing the virtual queue, the system utility maximization problem was equivalently decomposed into three subproblems. A distributed resource allocation scheme was proposed for the mixed integer resource allocation problem. The delay-aware dynamic resource management for multi-service in HSR communication system was investigated, which mainly concerned on power control along the time and the resource allocation of the services [17]. The uplink system capacity of HSR communications with massive multiple input multiple output (MIMO) was derived in [18], where the co-located antennas at the center of the train and the distributed antennas uniformly placed among the train were considered, respectively.

Furthermore, the security performance of HSR wireless communication system is very important. In order to ensure the safe transmission of the train control information, the cryptographic safely code by secret key was applied to the safety protocol[19, 20]. However, if the codebook can be intercepted and there is the enough processing ability, the key based encryption technology is easy to be cracked [21]. The improvement of the security for the HSR wireless communication system has been extensively concerned. Physical layer security (PLS) has been considered as an effective method to improve the security of wireless communication system, which can be applied to HSR communication system. From the perspective of information theory, the essence of PLS is to maximize the system security rate [22]. The security rate of the legitimate users is defined as the rate of the legitimate users minus the maximum rate of the eavesdropping users. If the rate of the eavesdropping user is greater than that of the legitimate user, the rate of the legitimate user is zero. Artificial noise and MIMO are often used to improve the secrecy performance [23]. The transmission strategy of joint beamforming and artificial noise was discussed to improve the security of HSR wireless communication in [24]. The legitimate user was on the top of the train, and the eavesdropping user was stationary beside the railway. Multiple remote antenna units were connected to the central processing unit, and a 
cooperative distributed secure spatial modulation was established, which could improve the security performance of HSR communication system. A joint optimal secrecy capacity algorithm was proposed to maximize the system security capacity, and the eavesdropping users were also stationary [25].

In this paper, the secure transmission for HSR communication system is investigated. The contributions are mainly summarized as follows:

(1) The considered HSR wireless communication system includes a macro base station (MBS), B roadside base stations (RBS) and K vehicle stations (VSs) on the top of the train, and the eavesdropping user is a mobile UAV. The objective function is to maximize the sum of the minimum security rate in each time slot. The constraint conditions are the QoS, switch requirements, and total power constraint.

(2) The original optimization problem is mixed-integer and non-convex. The block coordinate descent (BCD) method is applied and the original problem can be decoupled into two sub-problems. The one is the joint BS-VS association problem, and the other is the power control one.

(3) The first subproblem of optimizing BS-VS association can be solved by the successive convex approximation (SCA) algorithm, and the second subproblem of optimizing transmit power can be solved by the Lagrangian dual method [26]. Simulation results verify the effectiveness of the proposed algorithm.

The rest of this paper is organized as follows. In Section 2, the secure HSR communication system model is introduced and the joint BS-VS association and power control problem is formulated. Then, we proposed an efficient iterative algorithm to maximize the minimum secrecy rate by using some convex optimization techniques in Section 3. Finally, numerical results show the effectiveness of the proposed solution in Section 4, and Section 5 concludes this paper.

\section{Methods}

\subsection{System model}

The architecture of a HSR wireless communication network is illustrated in Fig. 1. A heterogeneous cellular network consisting of a MBS and $B$ RBS is considered to communicate with $K$ single antenna VSs installed on the train roof, where all RBSs are deployed at regular intervals in the coverage area of MBS and all BSs are configured with single antenna. The set of BSs is defined as $\mathcal{B}=\{0,1, \ldots, B\}$ where 0 represents the MBS and $\{1,2, \ldots, B\}$ are RBSs, and the set of VSs is defined as $\mathcal{K}=\{1,2, \ldots, K\}$. Moreover, there is a malicious eavesdropping UAV equipped with single antenna in the cell, and its trajectory can be known [27].

It is assumed that the running time of the high-speed train in the cell of MBS is $T$, which is equally split into $N$ time slots for the sake of convenience in analyzing the system model. The set of time slots is defined as $\mathcal{N}=\{1,2, \ldots, N\}$. In each time slot, the BSs send the requested data to the VSs and each VS is assumed to only associate with a RBS or the MBS. Let $x_{i k}[n] \in\{0,1\}$ be a binary variable for VS $k \in \mathcal{K}$ at time slot $n \in \mathcal{N}$ to indicate VS-to-BS $i \in \mathcal{B}$ association, i.e., $x_{i k}[n]=1$ if VS $k$ is associated with BS $i$ and $x_{i k}[n]=0$ otherwise. Since each VS is only associated with one BS at each time slot, we have

$$
\left\{\begin{array}{l}
\sum_{i \in \mathcal{B}} x_{i k}[n]=1, \forall k \in \mathcal{K}, \forall n \in \mathcal{N}, \\
x_{i k}[n] \in\{0,1\}, \forall i \in \mathcal{B}, \forall k \in \mathcal{K}, \forall n \in \mathcal{N} .
\end{array}\right.
$$




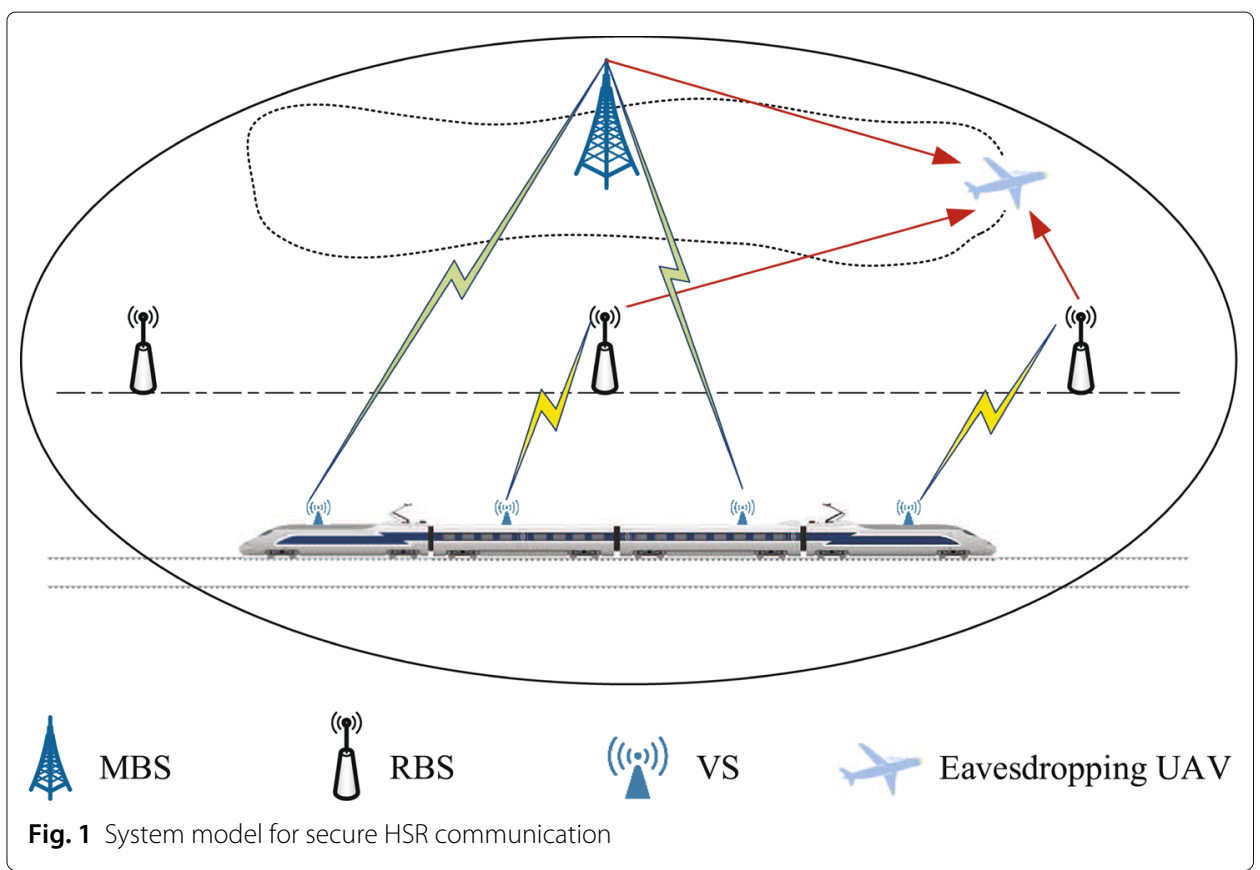

In order to avoid the high frequent switch and reduce associated overhead in the process of running, the VS is inclined to associate with the same BS in a period of consecutive time slots. Therefore, we give the following constraint

$$
\begin{aligned}
& \max \left\{\sum_{m=n}^{m=n+c} x_{0 k}[m], \ldots, \sum_{m=n}^{m=n+c} x_{B k}[m]\right\} \geq d, \forall k \in \mathcal{K}, \\
& \forall n \in\{1, \ldots, N-c\},
\end{aligned}
$$

where $c$ and $d$ are the constants to determine a tradeoff between low-switch and highperformance. $d$ is the maximum number of connections and $c$ is the number of time slots. The larger the number of connections in the fewer slots, the higher the performance of the system.

If VS $k$ is associated with BS $i$ at time slot $n$, the achievable data rate of the downlink transmission for VS $k$ is given by

$$
R_{k}[n]=\log _{2}\left(1+\frac{\sum_{i \in \mathcal{B}} x_{i k}[n] p_{i k}[n] h_{i k}[n]}{\sum_{j \in \mathcal{B}} \sum_{l \in \mathcal{K}, l \neq k} x_{j l}[n] p_{j l}[n] h_{j l}[n]+\sigma_{k}^{2}}\right),
$$

where $p_{i k}[n]$ is the transmit power from BS $i$ to VS $k$ at time slot $n, h_{i k}[n]$ is the downlink channel gain from BS $i$ to VS $k$ at time slot $n, \sum_{j \in \mathcal{B}} \sum_{l \in \mathcal{K}, l \neq k} x_{j l}[n] p_{j l}[n] h_{j l}[n]$ is the interference from BSs to all other VSs except $k$, and $\sigma_{k}^{2}$ is the noise power.

It is supposed that the eavesdropping UAV is located at the horizontal coordinate $\mathbf{w}_{u}[n]$ with the fixed altitude $H$ at time slot $n$, which are imperfectly known at the BSs. The location of BS $i \in \mathcal{B}$ is denoted as $\mathbf{l}_{i}$. We assume that UAV is also the network user and so it can be considered as the internal eavesdropping user. Since the propagation conditions between BSs and the eavesdropping UAV can be approximated as free space, we adopt the free-space path loss model to characterize the channel. Thus, the channel power gain between $\mathrm{BS} k$ and the UAV is dominated by the line-of-sight link and is written as 


$$
h_{i u}[n]=\frac{\varpi}{\left\|\mathbf{1}_{i}-\mathbf{w}_{u}[n]\right\|^{2}+H^{2}},
$$

where $\varpi=\xi_{i} \xi_{u}\left(\frac{\zeta}{4 \pi d_{0}}\right)^{2}$ denotes the channel power gain of BS-to-UAV link at the reference distance $d_{0}=1 \mathrm{~m}$, with $\zeta$ being the wavelength of the transmit signals, $\xi_{i}$ and $\xi_{u}$ being the transmitting gain of the antenna of BS $i$ and receiving gain of the antenna of $\mathrm{UAV}$, respectively. Therefore, the data rate for the eavesdropping UAV to eavesdrop the signal from the VS $k$ can be given as

$$
R_{k u}[n]=\log _{2}\left(1+\frac{\sum_{i \in \mathcal{B}} x_{i k}[n] p_{i k}[n] h_{i u}[n]}{\sum_{j \in \mathcal{B}} \sum_{l \in \mathcal{K}, l \neq k} x_{j l}[n] p_{j l}[n] h_{j u}[n]+\sigma_{u}^{2}}\right) .
$$

Since the VS $k \in \mathcal{K}$ does not know that the received signal is eavesdropped or not by the eavesdropping UAV, the secure communication between the associated BS and VS $k$ need to be guaranteed at any time slot. The secrecy rate for VS $k$ at time slot $n$ is given by

$$
R_{k}^{s}[n]=\left[R_{k}[n]-R_{k u}[n]\right]^{+},
$$

where $[a]^{+}=\max \{a, 0\}$.

\subsection{Problem formulation}

The aim of this paper is to maximize the sum of $N$ secrecy rates any of which is the minimum secrecy rate among all VSs at one time slot. We seek to optimize the BS-VS association $\boldsymbol{x} \triangleq\left\{x_{i k}[n], \forall i \in \mathcal{B}, \forall k \in \mathcal{K}, \forall n \in \mathcal{N}\right\}$ and power control $\boldsymbol{p} \triangleq$ $\left\{p_{i k}[n], \forall i \in \mathcal{B}, \forall k \in \mathcal{K}, \forall n \in \mathcal{N}\right\}$ under of guaranteeing QoS, less switch requirements and total power. The optimization problem can be formulated as

$$
\begin{aligned}
& \text { P1: } \max _{\boldsymbol{x}, \boldsymbol{p}} \frac{1}{N} \sum_{n=1}^{N} \min _{k \in \mathcal{K}} R_{k}^{s}[n] \\
& \text { s.t. } R_{k}[n] \geq \gamma_{k}^{\min }, \forall k \in \mathcal{K}, \forall n \in \mathcal{N}, \\
& \sum_{i \in \mathcal{B}} x_{i k}[n]=1, \forall k \in \mathcal{K}, \forall n \in \mathcal{N}, \\
& \quad x_{i k}[n] \in\{0,1\}, \forall i \in \mathcal{B}, \forall k \in \mathcal{K}, \forall n \in \mathcal{N}, \\
& \quad \max \left\{\left\{\sum_{m=n}^{m=n+c} x_{0 k}[m], \ldots, \sum_{m=n}^{m=n+c} x_{B k}[m]\right\} \geq d,\right. \\
& \forall k \in \mathcal{K}, \forall n \in\{1, \ldots, N-c\}, \\
& \sum_{k \in \mathcal{K}} x_{i k}[n] p_{i k}[n] \leq p_{i}^{\max }, \forall i \in \mathcal{B}, \forall n \in \mathcal{N},
\end{aligned}
$$

where $\gamma_{k}^{\min }$ is the minimum QoS requirement for VS $k \in \mathcal{K}$ and $p_{i}^{\max }$ is the peak transmit power of BS $i \in \mathcal{B}$.

The joint BS-VS association and power control problem in $P 1$ have some challenges to solve, since two key variables are coupled in $P 1$ and $P 1$ is in a mixed-integer and non-convex form. To solve $P 1$, we apply the BCD method and decouple $P 1$ into two subproblems. We solve the first subproblem of optimizing BS-VS association by applying the SCA technique, and then leverage the lagrangian dual method to solve the second subproblem of optimizing transmit power. 


\section{Proposed security rate maximization algorithm for HSR communication systems}

\subsection{BS-VS association subproblem}

When the power control vector $\boldsymbol{p}$ is fixed, the optimization problem $P 1$ can be rewritten as

$$
\begin{aligned}
& P 2: \max _{x} \frac{1}{N} \sum_{n=1}^{N} \min _{k \in \mathcal{K}} R_{k}^{s}[n] \\
& \text { s.t. (7b), (7c), (7d), (7e). }
\end{aligned}
$$

To begin with, $P 2$ is reformulated as $P 2.1$ by introducing the auxiliary variables $\chi[n], n \in \mathcal{N}$ and slacking the constraint (7d).

$$
\begin{aligned}
P 2.1: & \max _{x, \chi[n]} \frac{1}{N} \sum_{n=1}^{N} \chi[n] \\
\text { s.t. } & R_{k}[n]-R_{k u}[n] \geq \chi[n], \forall k \in \mathcal{K}, \forall n \in \mathcal{N}, \\
& \chi[n] \geq 0, \forall n \in \mathcal{N}, \\
& x_{i k}[n] \in[0,1], \forall i \in \mathcal{B}, \forall k \in \mathcal{K}, \forall n \in \mathcal{N}, \\
& \text { (7b), (7c), (7e). }
\end{aligned}
$$

In order to ensure the associated solutions are binary, we introduce the auxiliary variables $\mathbf{y} \triangleq\left\{y_{i k}[n], \forall i \in \mathcal{B}, \forall k \in \mathcal{K}, \forall n \in \mathcal{N}\right\}$ and give the following constraints.

$$
\left\{\begin{array}{l}
x_{i k}[n]\left(1-y_{i k}[n]\right)=0, \forall i \in \mathcal{B}, \forall k \in \mathcal{K} \\
x_{i k}[n]=y_{i k}[n], \forall i \in \mathcal{B}, \forall k \in \mathcal{K}
\end{array}\right.
$$

The constraint (10) is transformed into a penalty item to be added to the objective function [28]. Then, we get the following problem.

$$
\begin{gathered}
P 2.2: \max _{x, \chi[n]} \sum_{n=1}^{N}\left(\frac{\chi[n]}{N}-\omega_{n} \sum_{i \in \mathcal{B}} \sum_{k \in \mathcal{K}}\left(\left(x_{i k}[n]-y_{i k}[n]\right)^{2}\right.\right. \\
\left.\left.\quad+\left(x_{i k}[n]\left(1-y_{i k}[n]\right)\right)^{2}\right)\right) \\
\text { s.t. (9b), (9c), (9d), (7b), (7c), (7e), }
\end{gathered}
$$

where $\omega_{n}$ is penalty factor for $\forall n \in \mathcal{N}$.

To solve problem $P 2.2$, we propose the double-layer iterative algorithm where $y$ with the fixed $x$ and $\chi[n]$ is optimized in outer layer and the problem $P 2.2$ with $y$ is solved in inner layer. In outer layer, the optimal solution $y^{*}$ is obtained by solving the unconstrained optimization problem, as follows:

$$
\begin{aligned}
P 2.3: \max _{y} \phi & =\sum_{n=1}^{N}\left(\frac{\chi[n]}{N}-\omega_{n} \sum_{i \in \mathcal{B}} \sum_{k \in \mathcal{K}}\left(\left(x_{i k}[n]-y_{i k}[n]\right)^{2}\right.\right. \\
& \left.\left.+\left(x_{i k}[n]\left(1-y_{i k}[n]\right)\right)^{2}\right)\right) .
\end{aligned}
$$

By deriving the first-order derivative of problem $P 2.3$ with respect to $y_{i k}[n]$, the following equation can be obtained as

$$
\frac{\partial \phi}{\partial y_{i k}[n]}=2 \omega_{n}\left(x_{i k}[n]-y_{i k}[n]\right)+2 \omega_{n} x_{i k}^{2}[n]\left(1-y_{i k}[n]\right)=0 .
$$




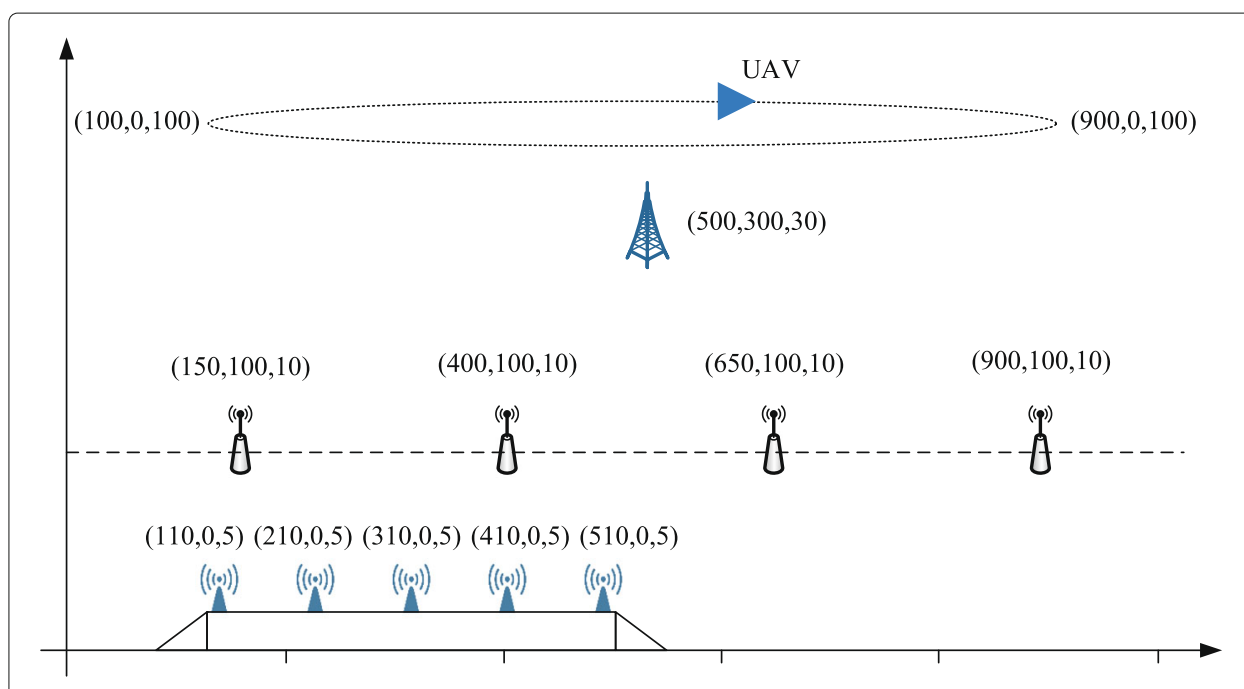

Fig. 2 The deployment locations of all BSs and VSs

Then, the optimal solution of $\mathbf{y}$ is obtained as

$$
y_{i k}^{*}[n]=\frac{x_{i k}[n]\left(1+x_{i k}[n]\right)}{1+x_{i k}^{2}[n]},
$$

for $\forall i \in \mathcal{B}, \forall k \in \mathcal{K}, \forall n \in \mathcal{N}$.

In inner layer, problem $P 2.1$ with the fixed $\mathbf{y}$ is non-convex due to constraint (9b). $R_{k}[n]-R_{k u}[n]$ is rewritten as

$$
\begin{aligned}
R_{k}[n]-R_{k u}[n] & =\left(\log _{2}\left(\Delta_{1}(\mathbf{x})+\sigma_{k}^{2}\right)+\log _{2}\left(\Delta_{4}(\mathbf{x})+\sigma_{u}^{2}\right)\right) \\
& -\left(\log _{2}\left(\Delta_{3}(\mathbf{x})+\sigma_{u}^{2}\right)+\log _{2}\left(\Delta_{2}(\mathbf{x})+\sigma_{k}^{2}\right)\right) \\
& \triangleq f_{1}(\mathbf{x})-g_{1}(\mathbf{x}),
\end{aligned}
$$

where $\Delta_{1}(\mathbf{x})=\sum_{j \in \mathcal{B}} \sum_{l \in \mathcal{K}} x_{j l}[n] p_{j l}[n] h_{j l}[n], \Delta_{2}(\mathbf{x})=\sum_{j \in \mathcal{B}} \sum_{l \in \mathcal{K}, l \neq k} x_{j l}[n] p_{j l}[n] h_{j l}[n], \Delta_{3}(\mathbf{x})=$ $\sum_{j \in \mathcal{B}} \sum_{l \in \mathcal{K}} x_{j l}[n] p_{j l}[n] h_{j u}[n]$, and $\Delta_{4}(\mathbf{x})=\sum_{j \in \mathcal{B}} \sum_{l \in \mathcal{K}, l \neq k} x_{j l}[n] p_{j l}[n] h_{j u}[n]$.

Since $f_{1}$ and $g_{1}$ are concave functions, $f_{1}(\mathbf{x})-g_{1}(\mathbf{x})$ is a subtraction form of two concave functions. We adopt the SCA technique to re-express $R_{k}[n]-R_{k u}[n]$ in the $t+1$-th iteration as

$$
\begin{aligned}
& R_{k}[n]-R_{k u}[n] \\
& \geq f_{1}(\mathbf{x})-\left(g_{1}\left(\mathbf{x}^{t}\right)+\nabla g_{1}\left(\mathbf{x}^{t}\right)^{T}\left(\mathbf{x}-\mathbf{x}^{t}\right)\right) \\
& \triangleq f_{1}(\mathbf{x})-\left(\log _{2}\left(\Delta_{3}\left(\mathbf{x}^{t}\right)+\sigma_{u}^{2}\right)+\log _{2}\left(\Delta_{2}\left(\mathbf{x}^{t}\right)+\sigma_{k}^{2}\right)\right. \\
& \left.+\frac{\Delta_{3}(\mathbf{x})-\Delta_{3}\left(\mathbf{x}^{t}\right)}{\ln 2\left(\Delta_{3}\left(\mathbf{x}^{t}\right)+\sigma_{u}^{2}\right)}+\frac{\Delta_{2}(\mathbf{x})-\Delta_{2}\left(\mathbf{x}^{t}\right)}{\ln 2\left(\Delta_{2}\left(\mathbf{x}^{t}\right)+\sigma_{k}^{2}\right)}\right) .
\end{aligned}
$$

Constraint (9b) is transformed as

$$
\begin{aligned}
& f_{1}(\mathbf{x})-\left(\log _{2}\left(\Delta_{3}\left(\mathbf{x}^{t}\right)+\sigma_{u}^{2}\right)+\log _{2}\left(\Delta_{2}\left(\mathbf{x}^{t}\right)+\sigma_{k}^{2}\right)\right. \\
& \left.+\frac{\Delta_{3}(\mathbf{x})-\Delta_{3}\left(\mathbf{x}^{t}\right)}{\ln 2\left(\Delta_{3}\left(\mathbf{x}^{t}\right)+\sigma_{u}^{2}\right)}+\frac{\Delta_{2}(\mathbf{x})-\Delta_{2}\left(\mathbf{x}^{t}\right)}{\ln 2\left(\Delta_{2}\left(\mathbf{x}^{t}\right)+\sigma_{k}^{2}\right)}\right)-\chi[n] \geq 0 .
\end{aligned}
$$

Constraint (7b) is rewritten as

$$
\Delta_{1}(\mathbf{x})-2^{\gamma_{k}^{\min }} \Delta_{2}(\mathbf{x})-\left(2^{\gamma_{k}^{\min }}-1\right) \sigma_{k}^{2} \geq 0 .
$$


Since the left item of constraint $(7 e)$ is convex, we introduce the function $f_{2}\left(\left(x_{i}\right)_{i \in \mathcal{B}}\right)$

$$
f_{2}\left(\left(x_{i}\right)_{i \in \mathcal{B}}\right)=\ln \left(\sum_{i \in \mathcal{B}} \exp \left(x_{i}\right)\right)
$$

with inequality $\max \left\{\left(x_{i}\right)_{i \in \mathcal{B}}\right\} \leq f_{2}\left(\left(x_{i}\right)_{i \in \mathcal{B}}\right) \leq \max \left\{\left(x_{i}\right)_{i \in \mathcal{B}}\right\}+\ln B$, and (7e) can be approximate to

$$
\begin{aligned}
& \max \left\{\left(\sum_{m=n}^{m=n+c} x_{i k}[m]\right)_{i \in \mathcal{B}}\right\} \\
& \geq f_{2}\left(\left(\sum_{m=n}^{m=n+c} x_{i k}[m]\right)_{i \in \mathcal{B}}\right)-\ln B \geq d .
\end{aligned}
$$

We adopt the SCA technique to re-express the above inequality by using the first order Taylor expansion of $f_{2}$ around a feasible point $\left\{x_{i k}[n]\right\}$ at the $(\mathrm{t}+1)$-th iteration. Constraint (7e) is re-expressed as (21).

$$
\begin{aligned}
& \frac{\sum_{i \in \mathcal{B}}\left(\sum_{m=n}^{m=n+c} x_{i k}[m]\right) \exp \left(\sum_{m=n}^{m=n+c} x_{i k}^{t}[m]\right)}{\sum_{i \in \mathcal{B}} \exp \left(\sum_{m=n}^{m=n+c} x_{i k}^{t}[m]\right)} \\
& -\frac{\sum_{i \in \mathcal{B}}\left(\sum_{m=n}^{m=n+c} x_{i k}^{t}[m]\right) \exp \left(\sum_{m=n}^{m=n+c} x_{i k}^{t}[m]\right)}{\sum_{i \in \mathcal{B}} \exp \left(\sum_{m=n}^{m=n+c} x_{i k}^{t}[m]\right)}-\ln B \geq d .
\end{aligned}
$$

Problem $P 2.2$ is reformulated as

$$
\begin{gathered}
P 2.4: \max _{x, \chi[n]} \sum_{n=1}^{N}\left(\frac{\chi[n]}{N}-\omega_{n} \sum_{i \in \mathcal{B}} \sum_{k \in \mathcal{K}}\left(\left(x_{i k}[n]-y_{i k}[n]\right)^{2}\right.\right. \\
\left.\left.+\left(x_{i k}[n]\left(1-y_{i k}[n]\right)\right)^{2}\right)\right)
\end{gathered}
$$

s.t. (17), (9c), (9d), (18), (7c), (21),

which is a convex optimization problem.

\subsection{BS transmit power subproblem}

When the BS-VS association vector $\mathbf{x}$ is given, $P 1$ can be split to $N$ subproblems, which is equivalently formulated as

$$
\begin{aligned}
& P 3: \max _{\boldsymbol{p}_{k}} \min _{k \in \mathcal{K}}\left[R_{k}-R_{k u}\right]^{+} \\
& \text {s.t. } R_{k} \geq \gamma_{k}^{\min }, \forall k \in \mathcal{K}, \\
& \sum_{k \in \mathcal{K}_{i}} p_{b_{k} k} \leq p_{i}^{\max }, \forall i \in \mathcal{B},
\end{aligned}
$$

where the symbol $[n]$ indicating time slot $n$ is omitted for the stake of convenience.

To convexify the objective function, we introduce two kinds of auxiliary variables $\rho$ and $\tau_{k}, k \in \mathcal{K}$ and $P 3$ is reformulated as

$$
\begin{aligned}
\text { P3.1 } & \max _{\boldsymbol{p}_{k}, \rho, \boldsymbol{\tau}} \rho \\
\text { s.t. } & R_{k}-\tau_{k} \geq \rho, \forall k \in \mathcal{K}, \\
& R_{k u} \leq \tau_{k}, \forall k \in \mathcal{K}, \\
& \rho \geq 0, \tau_{k}>0, \forall k \in \mathcal{K}, \\
& \text { (23b), (23c). }
\end{aligned}
$$


Since (24b) is non-convex, we introduce the inequality

$$
\log _{2}(1+\eta) \geq-\frac{\theta}{\eta}+\vartheta
$$

with

$$
\left\{\begin{array}{l}
\theta=\frac{\bar{\eta}^{2}}{1+\bar{\eta}} \\
\vartheta=\frac{\bar{\eta}}{1+\bar{\eta}}+\log _{2}(1+\bar{\eta})
\end{array}\right.
$$

where the inequality holds if $\eta=\bar{\eta}$, and get that

$$
R_{k} \geq-\theta_{k}^{t}\left(\frac{\sum_{l \in \mathcal{K}, l \neq k} p_{b_{l} l} h_{b_{l} l}+\sigma_{k}^{2}}{p_{b_{k} k} h_{b_{k} k}}\right)+\vartheta_{k}^{t} .
$$

Thus, constraint (24b) is rewritten as

$$
\theta_{k}^{t}\left(\sum_{l \in \mathcal{K}, l \neq k} p_{b_{l} l} h_{b_{l} l}+\sigma_{k}^{2}\right)+\left(\rho+\tau_{k}-\vartheta_{k}^{t}\right) p_{b_{k} k} h_{b_{k} k} \leq 0 .
$$

The second item in above inequality is coupled with respect to the optimization variables. Its first order Taylor expansion around a feasible point $\left(\rho^{t}, \tau_{k}^{t}, p_{b_{k} k}^{t}\right)$ at the $(t+1)$-th iteration is given as

$$
\begin{aligned}
& \left(\rho+\tau_{k}-\vartheta_{k}^{t}\right) p_{b_{k} k} h_{b_{k} k} \\
& =\frac{\left(\rho+\tau_{k}-\vartheta_{k}^{t}+p_{b_{k} k} h_{b_{k} k}\right)^{2}}{4}-\frac{\left(\rho+\tau_{k}-\vartheta_{k}^{t}-p_{b_{k} k} h_{b_{k} k}\right)^{2}}{4} \\
& \leq \frac{\left(\rho+\tau_{k}-\vartheta_{k}^{t}+p_{b_{k} k} h_{b_{k} k}\right)^{2}}{4}+\frac{\left(\rho^{t}+\tau_{k}^{t}-\vartheta_{k}^{t}-p_{b_{k} k}^{t} h_{b_{k} k}\right)^{2}}{4} \\
& -\frac{\left(\rho^{t}+\tau_{k}^{t}-\vartheta_{k}^{t}-p_{b_{k} k}^{t} h_{b_{k} k}\right)\left(\rho+\tau_{k}-\vartheta_{k}^{t}-p_{b_{k} k} h_{b_{k} k}\right)}{2} \\
& =\frac{\left(\rho+\tau_{k}-\vartheta_{k}^{t}+p_{b_{k} k} h_{b_{k} k}\right)^{2}}{4}+\frac{\Theta_{k}^{2}}{4}-\frac{\Theta_{k}\left(\rho+\tau_{k}-\vartheta_{k}^{t}-p_{b_{k} k} h_{b_{k} k}\right)}{2} .
\end{aligned}
$$

According to (29), inequality (24b) can be derived as a further tight constraint (30) at the $(t+1)$-th iteration.

$$
\begin{aligned}
& \theta_{k}^{t}\left(\sum_{l \in \mathcal{K}, l \neq k} p_{b_{l} l} h_{b_{l} l}+\sigma_{k}^{2}\right)+\frac{\left(\rho+\tau_{k}-\vartheta_{k}^{t}+p_{b_{k} k} h_{b_{k} k}\right)^{2}}{4}+\frac{\Theta_{k}^{2}}{4} \\
& -\frac{\Theta_{k}\left(\rho+\tau_{k}-\vartheta_{k}^{t}-p_{b_{k} k} h_{b_{k} k}\right)}{2} \leq 0 \\
\Rightarrow & \frac{\Theta_{k}\left(\rho+\tau_{k}-p_{b_{k} k} h_{b_{k} k}\right)}{2}-\theta_{k}^{t} \sum_{l \in \mathcal{K}, l \neq k} p_{b_{l} l} h_{b_{l} l}-\frac{\left(\rho+\tau_{k}-\vartheta_{k}^{t}+p_{b_{k} k} h_{b_{k} k}\right)^{2}}{4} \\
& -\left(\theta_{k}^{t} \sigma_{k}^{2}+\frac{\Theta_{k} \vartheta_{k}^{t}}{2}+\frac{\Theta_{k}^{2}}{4}\right) \geq 0 \\
\Rightarrow & \frac{\Theta_{k}\left(\rho+\tau_{k}-p_{b_{k} k} h_{b_{k} k}\right)}{2}-\theta_{k}^{t} \sum_{l \in \mathcal{K}, l \neq k} p_{b_{l} l} h_{b_{l} l}-\frac{\left(\rho+\tau_{k}-\vartheta_{k}^{t}+p_{b_{k} k} h_{b_{k} k}\right)^{2}}{4} \\
& -\Psi_{k} \geq 0 .
\end{aligned}
$$


Constraint (24c) is non-convex and expressed as

$$
R_{k u}=\log _{2}\left(1+\frac{p_{b_{k} k} h_{b_{k} u}}{\sum_{l \in \mathcal{K}, l \neq k} p_{b_{l}} l_{b_{l} u}+\sigma_{u}^{2}}\right) \leq \tau_{k} .
$$

To convexify (24c), the first order Taylor expansion of $2^{\tau_{k}}-1$ around a feasible point $\tau_{k}$ at the $(t+1)$-th iteration is given as

$$
2^{\tau_{k}}-1 \geq 2^{\tau_{k}^{t}}+2^{\tau_{k}^{t}} \ln 2\left(\tau_{k}-\tau_{k}^{t}\right)-1
$$

Thus, (24c) is converted into

$$
\frac{p_{b_{k} k} h_{b_{k} u}}{\sum_{l \in \mathcal{K}, l \neq k} p_{b_{l} l} h_{b_{l} u}+\sigma_{u}^{2}} \leq 2^{\tau_{k}^{t}}\left(1-\ln 2 \tau_{k}^{t}\right)-1+2^{\tau_{k}^{t}} \ln 2 \tau_{k} .
$$

Letting $\Gamma_{k}=2^{\tau_{k}^{t}}\left(1-\ln 2 \tau_{k}^{t}\right)-1$ and $\Xi_{k}=2^{\tau_{k}^{t}} \ln 2$, we have

$$
p_{b_{k} k} h_{b_{k} u} \leq\left(\Gamma_{k}+\Xi_{k} \tau_{k}\right)\left(\sum_{l \in \mathcal{K}, l \neq k} p_{b_{l} l} h_{b_{l} u}+\sigma_{u}^{2}\right) .
$$

The right item of inequality (34) is coupled with respect to the optimization variables $\tau_{k}$ and $p_{b_{l} l}$. We derive its first order Taylor expansion around a feasible point $\left(\tau_{k}, p_{b_{k} k}\right)$ at the $(t+1)$-th iteration, which is similar to the process of (29). The lower bound of the right item of inequality (34) is given as (35).

$$
\begin{aligned}
& \left(\Gamma_{k}+\Xi_{k} \tau_{k}\right)\left(\sum_{l \in \mathcal{K}, l \neq k} p_{b_{l} l} h_{b_{l} u}+\sigma_{u}^{2}\right)=\frac{\left(\Gamma_{k}+\Xi_{k} \tau_{k}+\sum_{l \in \mathcal{K}, l \neq k} p_{b_{l} l} h_{b_{l} u}+\sigma_{u}^{2}\right)^{2}}{4} \\
& -\frac{\left(\Gamma_{k}+\Xi_{k} \tau_{k}-\sum_{l \in \mathcal{K}, l \neq k} p_{b_{l} l} h_{b_{l} u}+\sigma_{u}^{2}\right)^{2}}{4} \\
& \geq \frac{\left(\Gamma_{k}+\Xi_{k} \tau_{k}^{t}+\sum_{l \in \mathcal{K}, l \neq k} p_{b_{l} l}^{t} h_{b_{l} u}+\sigma_{u}^{2}\right)\left(\Gamma_{k}+\Xi_{k} \tau_{k}+\sum_{l \in \mathcal{K}, l \neq k} p_{b_{l} l} h_{b_{l} u}+\sigma_{u}^{2}\right)}{2} \\
& -\frac{\left(\Gamma_{k}+\Xi_{k} \tau_{k}^{t}+\sum_{l \in \mathcal{K}, l \neq k} p_{b_{l} l}^{t} h_{b_{l} u}+\sigma_{u}^{2}\right)^{2}}{4}-\frac{\left(\Gamma_{k}+\Xi_{k} \tau_{k}-\sum_{l \in \mathcal{K}, l \neq k} p_{b_{l} l} h_{b_{l} u}+\sigma_{u}^{2}\right)^{2}}{4} \\
& -\frac{\Phi_{k}\left(\Gamma_{k}+\Xi_{k} \tau_{k}+\sum_{l \in \mathcal{K}, l \neq k} p_{b_{l} l} h_{b_{l} u}+\sigma_{u}^{2}\right)}{2}-\frac{\Phi_{k}^{2}}{4} \\
& \left.\quad \sum_{l \in \mathcal{K}, l \neq k} p_{b_{l} l} h_{b_{l} u}+\sigma_{u}^{2}\right)^{2} \\
& 4
\end{aligned}
$$




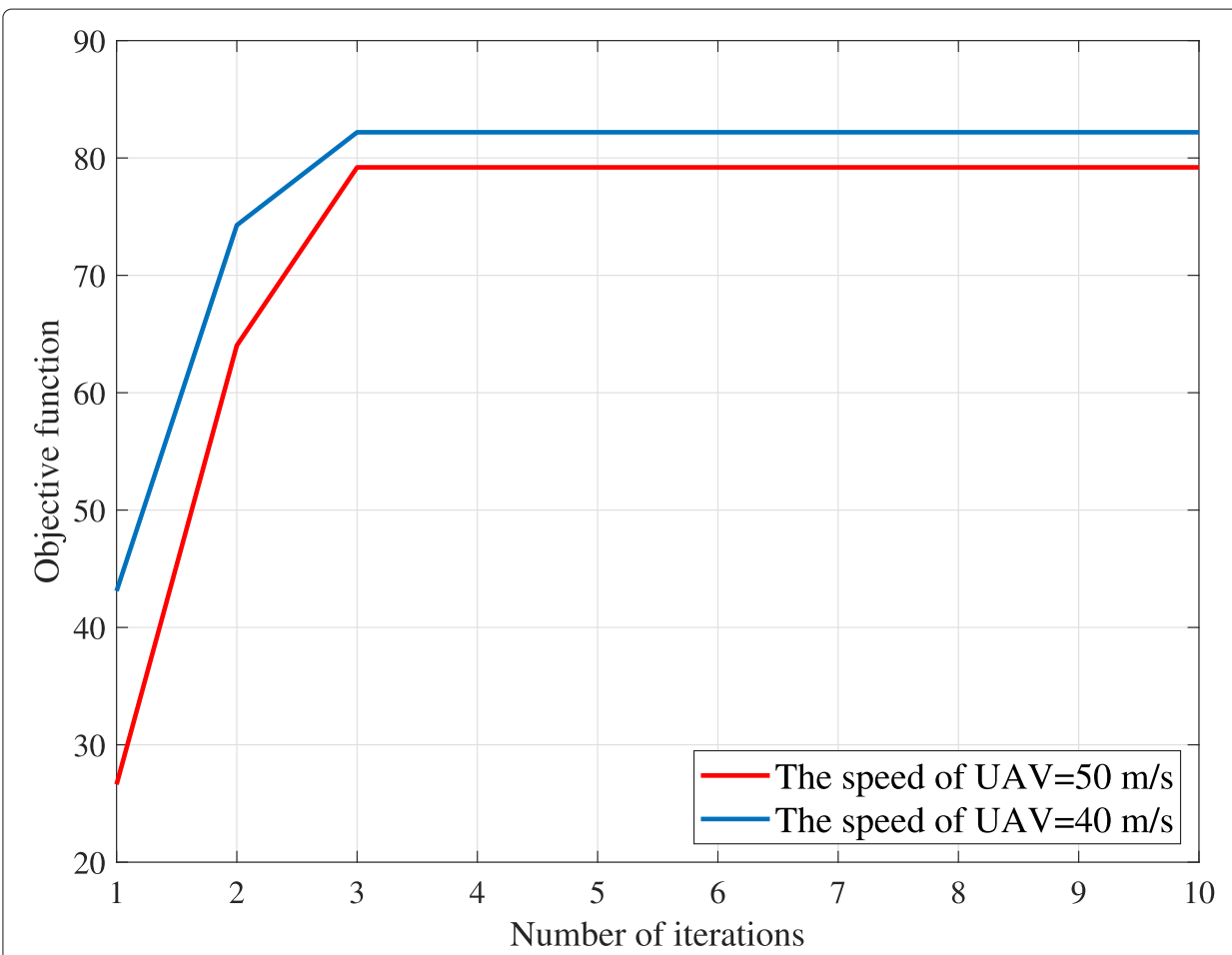

Fig. 3 BS-VS association performance with given power versus different flight speeds of UAV

According to (35), inequality (24c) can be derived as a further tight constraint (36) at the $(t+1)$-th iteration.

$$
\begin{aligned}
& p_{b_{k} k} h_{b_{k} u} \leq \frac{\Phi_{k}\left(\Gamma_{k}+\Xi_{k} \tau_{k}+\sum_{l \in \mathcal{K}, l \neq k} p_{b_{l} l} h_{b_{l} u}+\sigma_{u}^{2}\right)}{2}-\frac{\Phi_{k}^{2}}{4} \\
& -\frac{\left(\Gamma_{k}+\Xi_{k} \tau_{k}-\sum_{l \in \mathcal{K}, l \neq k} p_{b_{l} l} h_{b_{l} u}+\sigma_{u}^{2}\right)^{2}}{4} \\
& \Rightarrow \frac{\Phi_{k} \Xi_{k} \tau_{k}}{2}+\frac{\Phi_{k} \sum_{l \in \mathcal{K}, l \neq k} p_{b_{l} l} h_{b_{l} u}}{2}-\frac{\left(\Gamma_{k}+\Xi_{k} \tau_{k}-\sum_{l \in \mathcal{K}, l \neq k} p_{b_{l} l} h_{b_{l} u}+\sigma_{u}^{2}\right)^{2}}{4} \\
& -p_{b_{k} k} h_{b_{k} u}+\frac{\Phi_{k}\left(\Gamma_{k}+\sigma_{u}^{2}\right)}{2}-\frac{\Phi_{k}^{2}}{4} \geq 0 \\
& \Rightarrow \frac{\Phi_{k} \Xi_{k} \tau_{k}}{2}+\frac{\Phi_{k} \sum_{l \in \mathcal{K}, l \neq k} p_{b_{l} l} h_{b_{l} u}}{2}-\frac{\left(\Gamma_{k}+\Xi_{k} \tau_{k}-\sum_{l \in \mathcal{K}, l \neq k} p_{b_{l} l} h_{b_{l} u}+\sigma_{u}^{2}\right)^{2}}{4} \\
& -p_{b_{k} k} h_{b_{k} u}+\Upsilon_{k} \geq 0 .
\end{aligned}
$$

The constraint (23b) $R_{k} \geq \gamma_{k}^{\min }$ can be rewritten as

$$
p_{b_{k} k} h_{b_{k} k}-\left(2^{\gamma_{k}^{\min }}-1\right) \sum_{l \in \mathcal{K}, l \neq k} p_{b_{l}} h_{b_{l} l}-\left(2^{\gamma_{k}^{\min }}-1\right) \sigma_{k}^{2} \geq 0
$$


Therefore, problem $P 3.1$ is reformulated as

$$
\begin{aligned}
& \text { P3.2 : } \max _{\boldsymbol{p}_{k}, \rho, \boldsymbol{\tau}} \rho \\
& \text { s.t. (30), (36), (24d), (37), (23c). }
\end{aligned}
$$

In the following, we use the Lagrange dual technique to solve P3.2. The Lagrangian function of (38) is given as (39).

$$
\begin{aligned}
& \mathcal{L}(\mathbf{p}, \rho, \boldsymbol{\tau}, \boldsymbol{\lambda}, \boldsymbol{\mu}, \boldsymbol{\kappa}, \boldsymbol{v}) \\
& =\rho+\sum_{k \in \mathcal{K}} \lambda_{k}\left(\frac{\Theta_{k}\left(\rho+\tau_{k}-p_{b_{k}} h_{b_{k} k}\right)}{2}-\theta_{k}^{t} \sum_{l \in \mathcal{K}, l \neq k} p_{b_{l} l} h_{b_{l} l}\right. \\
& \left.-\frac{\left(\rho+\tau_{k}-\vartheta_{k}^{t}+p_{b_{k} k} h_{b_{k} k}\right)^{2}}{4}-\Psi_{k}\right)+\sum_{k \in \mathcal{K}} \mu_{k}\left(\frac{\Phi_{k} \Xi_{k} \tau_{k}}{2}+\frac{\Phi_{k} \sum_{l \in \mathcal{K}, l \neq k} p_{b_{l}} h_{b_{l} u}}{2}\right. \\
& \left.-\frac{\left(\Gamma_{k}+\Xi_{k} \tau_{k}-\sum_{l \in \mathcal{K}, l \neq k} p_{b_{l} l} h_{b_{l} u}+\sigma_{u}^{2}\right)^{2}}{4}-p_{b_{k} k} h_{b_{k} u}+\Upsilon_{k}\right)+\sum_{k \in \mathcal{K}} \kappa_{k}\left(p_{b_{k} k} h_{b_{k} k}\right. \\
& \left.-\left(2^{\gamma_{k}^{\min }}-1\right) \sum_{l \in \mathcal{K}, l \neq k} p_{b_{l} l} h_{b_{l} l}-\left(2^{\gamma_{k}^{\min }}-1\right) \sigma_{k}^{2}\right)+\sum_{i \in \mathcal{B}} v_{i}\left(p_{i}^{\max }-\sum_{k \in \mathcal{K}_{i}} p_{b_{k} k}\right) .
\end{aligned}
$$

where $\lambda \triangleq\left\{\lambda_{k}, k \in \mathcal{K}\right\}, \boldsymbol{\mu} \triangleq\left\{\mu_{k}, k \in \mathcal{K}\right\}, \boldsymbol{\kappa} \triangleq\left\{\kappa_{k}, k \in \mathcal{K}\right\}, \boldsymbol{\nu} \triangleq\left\{v_{i}, i \in \mathcal{B}\right\}$ are the Lagrangian multipliers.
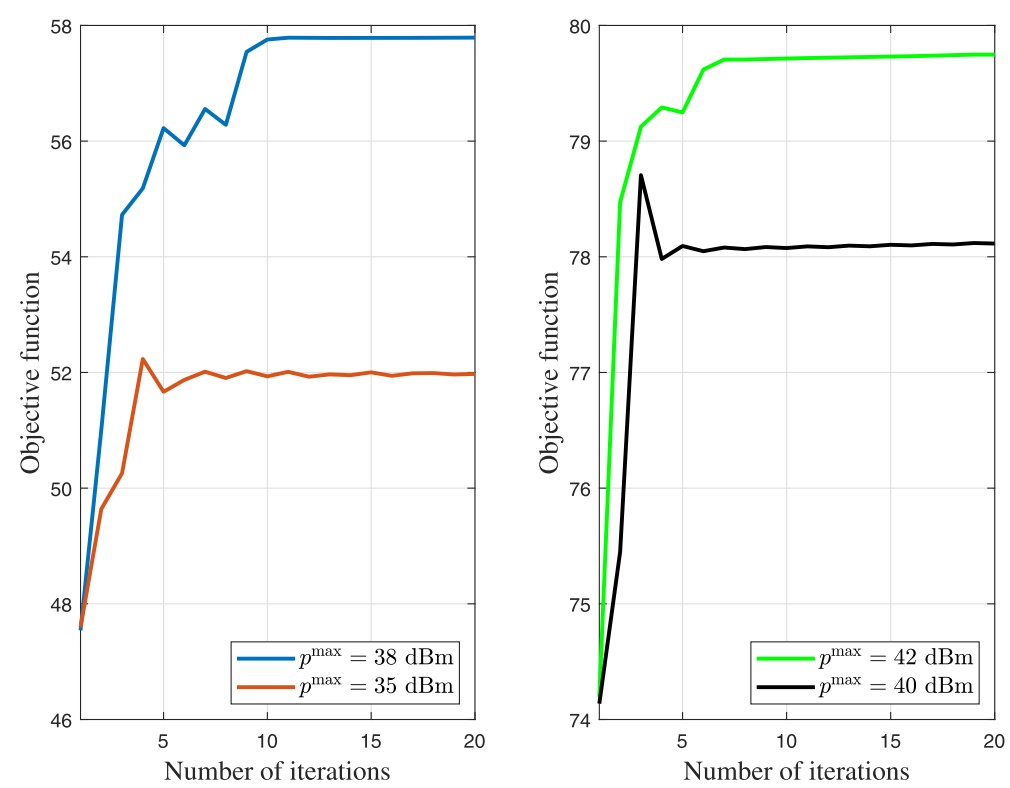

Fig. 4 The convergence behavior analysis of Algorithm 2 under the different maximum transmit power of BSs 
Then, the corresponding dual function is represented as

$$
\begin{aligned}
\Phi(\boldsymbol{\lambda}, \boldsymbol{\mu}, \boldsymbol{\kappa}, \boldsymbol{v})= & \min _{\mathbf{p} k, \rho, \boldsymbol{\tau}} \mathcal{L}\left(\mathbf{p}_{k}, \rho, \boldsymbol{\tau}, \boldsymbol{\lambda}, \boldsymbol{\mu}, \boldsymbol{\kappa}, \boldsymbol{v}\right) \\
& \text { s.t. } \rho \geq 0, \tau_{k} \geq 0
\end{aligned}
$$

Thus, the dual problem of $P 3.2$ is given by

$$
\begin{aligned}
& \text { P3.3 : } \max _{\lambda, \mu, \kappa, v} \Phi(\lambda, \mu, \kappa, v) \\
& \text { s.t. } \lambda_{k} \geq 0, \mu_{k} \geq 0, \kappa_{k} \geq 0, \forall k \in \mathcal{K}, \\
& \\
& v_{i} \geq 0, \forall i \in \mathcal{B} .
\end{aligned}
$$

The strong duality always exists since $P 3.2$ is a convex optimization problem. For any given $(\lambda, \mu, \kappa, v)$, the optimal solution $\left(\mathbf{p}^{*}, \rho^{*}, \boldsymbol{\tau}^{*}\right)$ can be obtained by the following theorems.

Theorem 1 For any given $(\lambda, \mu, \kappa, v)$, the optimal solution of power control for all VSs is given by (43).

Proof When $(\lambda, \mu, \kappa, v)$ is given, the Eq. 42 is obtained by deriving the first-order derivative of $\mathcal{L}$ with respect to $p_{b_{k} k}$ for $\forall k \in \mathcal{K}$. Thus, the optimal solution of power allocation for all VSs can be get by (43).

$$
\begin{aligned}
& \frac{\partial \mathcal{L}}{\partial p_{b_{k} k}}=-\frac{p_{b_{k} k} h_{b_{k} k}^{2} \lambda_{k}}{2}-\left(\sum_{l \in \mathcal{K}, l \neq k} \lambda_{l} \theta_{l}^{t}+\frac{\left(\Theta_{k}+\left(\rho+\tau_{k}-\vartheta_{k}^{t}\right)\right) \lambda_{k}}{2}\right) h_{b_{k} k} \\
& +\left(\sum_{l \in \mathcal{K}, l \neq k} \frac{\mu_{l}\left(\Phi_{l}+\left(\Gamma_{l}+\Xi_{l} \tau_{l}-\sum_{m \in \mathcal{K}, m \neq l, k} p_{b_{m} m} h_{b_{m} u}+\sigma_{u}^{2}\right)\right)}{2}-\mu_{k}\right) h_{b_{k} u} \\
& -\sum_{l \in \mathcal{K}, l \neq k} \frac{p_{b_{k} k} h_{b_{k} u}^{2} \mu_{l}}{2}+\left(\kappa_{k}-\sum_{l \in \mathcal{K}, l \neq k}\left(2^{\left.\left.\gamma_{l}^{\min }-1\right) \kappa_{l}\right)} h_{b_{k} k}-v_{i} \mathbb{I}_{k \in K_{i}}=0 .\right.\right. \\
& p_{b_{k} k}^{*}=\frac{\left(\sum_{l \in \mathcal{K}, l \neq k} \mu_{l}\left(\Phi_{l}+\left(\Gamma_{l}+\Xi_{l} \tau_{l}-\sum_{m \in \mathcal{K}, m \neq l, k} p_{b_{m} m} h_{b_{m} u}+\sigma_{u}^{2}\right)\right)-2 \mu_{k}\right) h_{b_{k} u}}{\lambda_{k} h_{b_{k} k}^{2}+\sum_{l \in \mathcal{K}, l \neq k} \mu_{l} h_{b_{k} u}^{2}} \\
& +\frac{\left(2 \kappa_{k}-\sum_{l \in \mathcal{K}, l \neq k} 2\left(2^{\gamma_{l}^{\min }}-1\right) \kappa_{l}-\left(\sum_{l \in \mathcal{K}, l \neq k} 2 \lambda_{l} \theta_{l}^{t}+\left(\Theta_{k}+\left(\rho+\tau_{k}-\vartheta_{k}^{t}\right)\right) \lambda_{k}\right)\right) h_{b_{k} k}}{\lambda_{k} h_{b_{k} k}^{2}+\sum_{l \in \mathcal{K}, l \neq k} \mu_{l} h_{b_{k} u}^{2}} \\
& +\frac{2 v_{i} \mathbb{I}_{k \in K_{i}}}{\lambda_{k} h_{b_{k} k}^{2}+\sum_{l \in \mathcal{K}, l \neq k} \mu_{l} h_{b_{k} u}^{2}} .
\end{aligned}
$$


Theorem 2 For any given $(\boldsymbol{\lambda}, \boldsymbol{\mu}, \boldsymbol{\kappa}, \boldsymbol{v})$, the optimal auxiliary variables $\rho^{*}$ and $\tau_{k}^{*}$ for any VS $k \in \mathcal{K}$ can be obtained by

$$
\left\{\begin{aligned}
\rho^{*} & =\frac{2+\sum_{k \in \mathcal{K}} \lambda_{k}\left(\Theta_{k}-\left(\tau_{k}-\vartheta_{k}^{t}+p_{b_{k}} h_{b_{k} k}\right)\right)}{\sum_{k \in \mathcal{K}} \lambda_{k}}, \\
\tau_{k}^{*} & =\frac{\sum_{k \in \mathcal{K}} \lambda_{k}\left(\Theta_{k}-\left(\rho-\vartheta_{k}^{t}+p_{b_{k} k} h_{b_{k} k}\right)\right)}{\sum_{k \in \mathcal{K}} \lambda_{k}+\sum_{k \in \mathcal{K}} \mu_{k} \Xi_{k}^{2}} \\
+ & \frac{\sum_{k \in \mathcal{K}} \mu_{k}\left(\Phi_{k} \Xi_{k}-\Xi_{k}\left(\Gamma_{k}-\sum_{l \in \mathcal{K}, l \neq k} p_{b_{l} l} h_{b_{l} u}+\sigma_{u}^{2}\right)\right)}{\sum_{k \in \mathcal{K}} \lambda_{k}+\sum_{k \in \mathcal{K}} \mu_{k} \Xi_{k}^{2}}
\end{aligned}\right.
$$

Proof: It is assumed that $(\lambda, \mu, \kappa, v)$ have been given. By deriving the first-order derivative of $\mathcal{L}$ with respect to $\rho$ and $\tau_{k}$ respectively, we can obtain the following equations

$$
\frac{\partial \mathcal{L}}{\partial \rho}=1+\sum_{k \in \mathcal{K}} \lambda_{k}\left(\frac{\Theta_{k}}{2}-\frac{\left(\rho+\tau_{k}-\vartheta_{k}^{t}+p_{b_{k} k} h_{b_{k} k}\right)}{2}\right)=0,
$$

and

$$
\begin{aligned}
& \frac{\partial \mathcal{L}}{\partial \tau_{k}}=\sum_{k \in \mathcal{K}} \lambda_{k}\left(\frac{\Theta_{k}}{2}-\frac{\left(\rho+\tau_{k}-\vartheta_{k}^{t}+p_{b_{k} k} h_{b_{k} k}\right)}{2}\right) \\
& +\sum_{k \in \mathcal{K}} \mu_{k}\left(\frac{\Phi_{k} \Xi_{k}}{2}-\frac{\Xi_{k}\left(\Gamma_{k}+\Xi_{k} \tau_{k}-\sum_{l \in \mathcal{K}, l \neq k} p_{b_{l} l} h_{b_{l} u}+\sigma_{u}^{2}\right)}{2}\right)=0 .
\end{aligned}
$$

Thus, the optimal solution $\rho^{*}$ and $\tau_{k}^{*}, k \in \mathcal{K}$ can be obtained by (44).

\subsection{Proposed iterative algorithm}

Algorithm 1 The BS-VS association algorithm for solving $P 2$.

1: Initialization: Initialize the associated vector $\mathbf{x}^{0} \triangleq\left\{x_{i k}^{0}[n], \forall i \in \mathcal{B}, \forall k \in \mathcal{K}, \forall n \in \mathcal{N}\right\}$.

Loop for $t_{1}=1,2, \cdots$,

2: Use the feasible point $\mathbf{x}^{t_{1}-1}$ to update the solution $\mathbf{y}^{t_{1}} \triangleq\left\{y_{i k}^{t_{1}}[n], \forall i \in \mathcal{B}, \forall k \in \mathcal{K}, \forall n \in\right.$ $\mathcal{N}\}$ from (14).

Loop for $t=1,2, \cdots$,

3: Constraint (17) is updated based on the feasible point $\mathbf{x}^{t-1}$.

4: Obtain the optimal BS-VS association solution $\mathbf{x}^{t}$ by solving the convex optimization problem $P 2.4$.

5: If the convergence holds, end loop and let $\mathbf{x}^{t_{1}}=\mathbf{x}^{t}$.

6: $t=t+1$

\section{End loop}

7: $t_{1}=t_{1}+1$

8: If the convergence holds, end loop and output the optimal solution $\mathbf{x}^{*}=\mathbf{x}^{t}$.

End loop

According to the aforementioned analysis, the proposed iterative algorithms are described in Algorithm 1 where the BS-VS association $\mathbf{x}$ with given $\mathbf{p}$ is optimized and in Algorithm 2 where the power control $\mathbf{p}$ with given $\mathbf{x}$ is solved. The joint optimization 

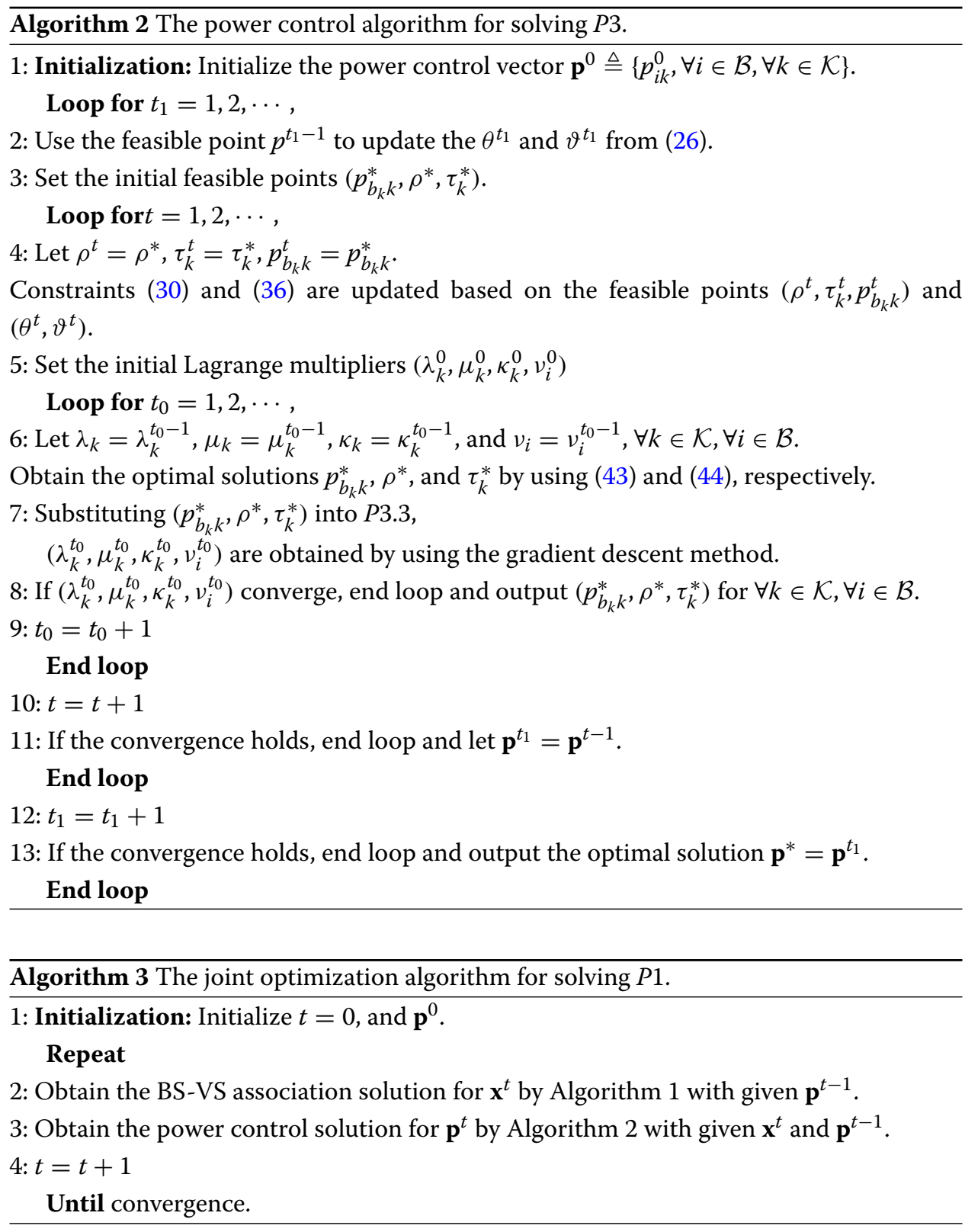

procedure is presented in Algorithm 3. In order to verify the convergence of Algorithm 3, we define $\phi_{1}(\mathbf{x}, \mathbf{p}), \phi_{2}(\mathbf{x}, \mathbf{p}), \phi_{2.1}(\mathbf{x}, \mathbf{p}), \phi_{2.2}(\mathbf{x}, \mathbf{p}), \phi_{3}(\mathbf{x}, \mathbf{p}), \phi_{3.1}(\mathbf{x}, \mathbf{p})$, and $\phi_{3.2}(\mathbf{x}, \mathbf{p})$ as the objective values of $P 1, P 2, P 2.1, P 2.2, P 3, P 3.1$, and P3.2. In step 4 of Algorithm 1, we can obtain the solution $\tilde{\mathbf{x}}^{t+1}$ to $P 2.4$ with a feasible point $\tilde{\mathbf{x}}^{t}$. As the iterative number increasing in step 3 and step 5 , the feasible point is updated and the feasible region is enlarged with the SCA method. When $\tilde{\mathbf{x}}^{t+1}=\tilde{\mathbf{x}}^{t}$, we have $\phi_{2.2}\left(\tilde{\mathbf{x}}^{t}, \tilde{\mathbf{x}}^{t+1}, \mathbf{p}^{t}\right) \leq \phi_{2.2}\left(\tilde{\mathbf{x}}^{t+1}, \tilde{\mathbf{x}}^{t+1}, \mathbf{p}^{t}\right)=$ $\phi_{2.1}\left(\mathbf{x}^{t+1}, \mathbf{p}^{t}\right)$, where $\mathbf{x}^{t+1}$ is the globally optimal solution for $P 2.1$ with fixed $\mathbf{p}^{t}$. Since $P 2.1$ is equivalent to $P 2$, the inequality $\phi_{2}\left(\mathbf{x}^{t}, \mathbf{p}^{t}\right) \leq \phi_{2}\left(\mathbf{x}^{t+1}, \mathbf{p}^{t}\right)$ holds. From step 6 to step 9 in Algorithm 2, the solution $\tilde{\mathbf{p}}^{t+1}$ to $P 3.2$ with a feasible point $\left(\tilde{\mathbf{p}}^{t}, \tilde{\rho}^{t}, \tilde{\boldsymbol{\tau}}^{t}\right)$ is obtained. Constraints (30) and (36) are relaxed by updating $\left(\tilde{\mathbf{p}}^{t}, \tilde{\rho}^{t}, \tilde{\boldsymbol{\tau}}^{t}\right)$ and $\left(\tilde{\theta}^{t}, \tilde{\vartheta}^{t}\right)$. When $\tilde{\mathbf{p}}^{t+1}=\tilde{\mathbf{p}}^{t}$, we have $\phi_{3.2}\left(\mathbf{x}^{t+1}, \tilde{\mathbf{p}}^{t+1}, \tilde{\mathbf{p}}^{t}\right) \leq \phi_{3.2}\left(\mathbf{x}^{t+1}, \tilde{\mathbf{p}}^{t+1}, \tilde{\mathbf{p}}^{t+1}\right)=\phi_{3.1}\left(\mathbf{x}^{t+1}, \mathbf{p}^{t+1}\right)$, where $\mathbf{p}^{t+1}$ is the globally optimal solution for $P 3.1$ with fixed $\mathbf{x}^{t+1}$. Since $P 3.1$ is equivalent to $P 3$, the 


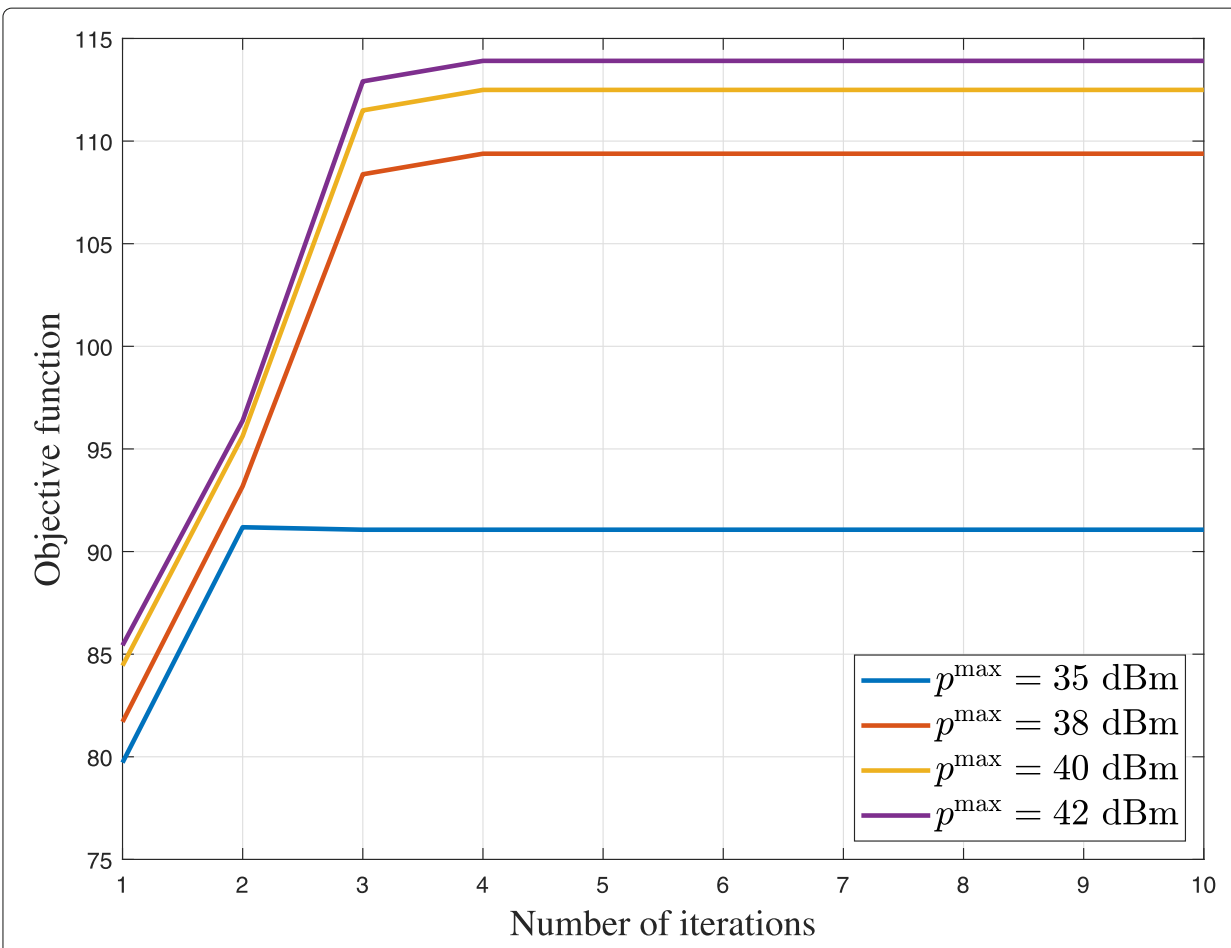

Fig. 5 The convergence behavior analysis of Algorithm 3 under the different maximum transmit power of BSs

inequality $\phi_{3}\left(\mathbf{x}^{t+1}, \mathbf{p}^{t}\right) \leq \phi_{3}\left(\mathbf{x}^{t+1}, \mathbf{p}^{t+1}\right)$ holds. In Algorithm 3 , the obtained solutions in step 2 and step 3 have the following relationship: $\phi_{1}\left(\mathbf{x}^{t}, \mathbf{p}^{t}\right)=\phi_{2}\left(\mathbf{x}^{t}, \mathbf{p}^{t}\right) \leq \phi_{2}\left(\mathbf{x}^{t+1}, \mathbf{p}^{t}\right)=$ $\phi_{3}\left(\mathbf{x}^{t+1}, \mathbf{p}^{t}\right) \leq \phi_{3}\left(\mathbf{x}^{t+1}, \mathbf{p}^{t+1}\right)=\phi_{1}\left(\mathbf{x}^{t+1}, \mathbf{p}^{t+1}\right)$. That is $\phi_{1}\left(\mathbf{x}^{t}, \mathbf{p}^{t}\right) \leq \phi_{1}\left(\mathbf{x}^{t+1}, \mathbf{p}^{t+1}\right)$. It is obviously obtained that $\phi_{1}$ is monotonically non-decreasing with respect to the iteration number and is upper bounded by a finite value. Therefore, Algorithm 3 is guaranteed to converge.

\section{Results and discussion}

\subsection{Parameters setting}

In this subsection, we present numerical results to validate our analysis and demonstrate the effectiveness of the proposed algorithms. We consider a railway segment of length 1 $\mathrm{km}$ where one MBS and $B=4$ RBSs are deployed. A HSR with $K=5 \mathrm{VSs}$ is running from point $(100,0)$ to point $(260,0)$ with the speed of $80 \mathrm{~m} / \mathrm{s}$ during $T=2 \mathrm{~s}$. The length of each time slot is set as $0.2 \mathrm{~s}$. The eavesdropping UAV have a fixed trajectory which is that the UAV with fixed $H=100$ travels back and forth between the starting point $(100,0,100)$ and ending point $(900,0,100)$ along the straight line at a uniform speed of $40 \mathrm{~m} / \mathrm{s}$. The air-to-air channel power gain is set as $\varpi=10^{-4}$ and the noise power is $\sigma_{k}^{2}=\sigma_{u}^{2}=-110 \mathrm{dBm}$. We consider the Rayleigh fading model between BS and VS. The path loss between MBS/RBS and VS is modeled as $P L^{M B S}=128.1+37.6 \log 10\left(d_{i k}[\mathrm{~km}]\right)$ and $P L^{R B S}=141.1+36.4 \log 10\left(d_{i k}[\mathrm{~km}]\right)$, where $d_{i k}$ is the distance (in $\mathrm{km}$ ) between MBS/RBS $i$ and VS $k$. The peak power budget of MBS/RBS is set to $p_{0}^{\max }=46 \mathrm{dBm}$ and $p_{i}^{\max }=30 \mathrm{dBm}, i \in \mathcal{B} /\{0\}$. Figure 2 gives the corresponding coordinate positions of all BSs and VSs. 


\subsection{Discussion}

Figure 3 shows the influence of the different flight speeds of UAV on the BS-VS association performance with the given power in Algorithm 1. It can be seen from Fig. 3 that for the given flight speed of UAV, when the number of iterations increases from 1 to 3 , the system performance gradually increases. When the number of iterations is greater than 3 , the system performance tends to be stable, which shows that Algorithm 1 has the good convergence and stability. It can also be seen from Fig. 3 that the flight speeds of UAV has an important impact on the system performance. The lower the flight speed of UAV, the higher the system performance. This is because the high flight speed of UAV increases the chance of eavesdropping VS.

Figure 4 gives the convergence performance of Algorithm 2 when the maximum transmission power of BS is different. As can be seen from Fig. 4, at first, the system performance gradually increases with the increase of iterations. When the number of iterations is between 3 and 10, the system performance jumps. For example, when Pmax = $35 \mathrm{dbm}$, the system performance after the fourth iteration is higher than that of the following iterations. When the number of iterations is more than 10 , the performance of Algorithm 2 tends to be stable. It can also be seen from Fig. 4 that the higher the power, the better the system performance.

Figure 5 shows the convergence performance of Algorithm 3 for the different maximum transmission power of BS. It can be seen from Fig. 5 that, with the increase of the number of the iterations, the system performance is monotonically increasing and then tends to be stable. When the maximum transmission power of BS is $35 \mathrm{dbm}$, the system performance tends to be stable after two iterations. When the maximum transmission power
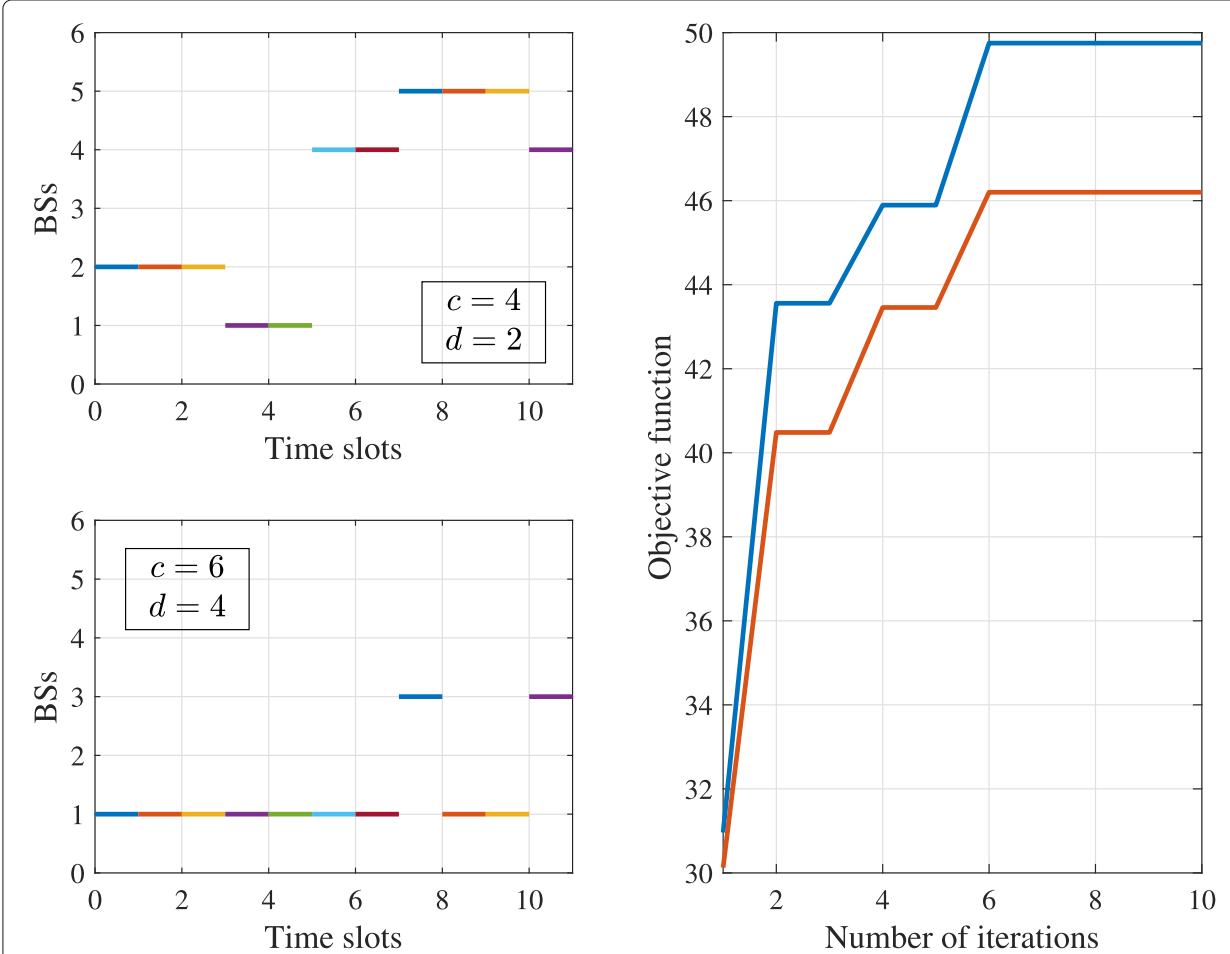

Fig. 6 BS-VS association with given power during T and the tradeoff between the optimization objective and low-switch by setting $c$ and $d$ 
of BS is $38 \mathrm{dbm}, 40 \mathrm{dbm}$, and $42 \mathrm{dbm}$, the system performance tends to be stable after four iterations. Similar to Fig. 4, the higher the maximum transmission power of BS, the better the system performance. When the maximum transmission power of BS is $42 \mathrm{dbm}$, the maximum system performance of Algorithm 2 in Fig. 4 is about $79.8 \mathrm{bit} / \mathrm{s} / \mathrm{Hz}$, while the maximum system performance of Algorithm 3 with the joint optimization in Fig. 5 is $114 \mathrm{bit} / \mathrm{s} / \mathrm{Hz}$. This is because Algorithm 3 in Fig. 5 jointly considers the power optimization and the BS-VS association, while Algorithm 2 in Fig. 4 only considers the power optimization for the given BS-VS association.

Figure 6 shows the connection state of VSs and the system performance with different $c$ and $d$. As can be seen from Fig. 6 , when $c=6$ and $d=4$, there is only one VS-BS association in the first seven time slots and it remains unchanged. In the 8th time slot, there are three VS-BS association. In the 9th and 10th time slots, there is only one VS-BS association. In the 11th time slot, there are three VS-BS association. It can be seen that when $c=$ $6, d=4$, the switch frequency is lower, while when $c=4, d=2$, the switch frequency is relatively high. It can be seen from the relationship between the system performance and the number of iterations in Fig. 6 that, when the switch frequency is low, the system performance will be reduced.

\section{Conclusion}

In this paper, the secure transmission for heterogeneous HSR communication system was studied, which contained a MBS, B RBSs, and K VSs on the top of the train, and a mobile eavesdropping UAV. Our goal was to maximize the sum of the minimum security rate of each time slot, and the constraint conditions contained the QoS, switch requirements, and the total power constraint. The original optimization problem was intractable to solve directly as it was mixed-integer and non-convex. The BCD method was applied, and the original problem could be decoupled into two sub-problems. The one was the joint BSVS association problem, and the other was the power control problem. The first subproblem of optimizing BS-VS association could be solved by the SCA algorithm, and the second sub-problem of optimizing transmit power could be solved by the Lagrangian dual method. In the future, we will consider the impact of beamforming and AN on the system security performance.

\footnotetext{
Abbreviations

HSR: High-speed railway; VS: Vehicle stations; UAV: Unmanned aerial vehicle; QoS: Quality of service; GSM-R: Global system for mobile communications for railway; BS: Base station; MRS: Mobile relay station; DoD: Direction of departure; CA: Co-located antennas; DA: Distributed antennas; AN: Artificial noise; MIMO: Multiple input multiple output; RAU: Remote antenna units; BCD: Block coordinate descent; SCA: Successive convex approximation; MBS: Macro base station; RBS: Roadside base station
}

Acknowledgements

Not applicable.

\section{Authors' contributions}

The research and the outcome of this specific publication are result of a long cooperation between the authors about fundamentals and applications of secure HSR communication systems. For the present manuscript, we make a contribution to joint optimization of BS-VS association and power control. All authors read and approved the final manuscript. 
PhD. Program of Traffic Information Engineering and Control in China Academy of Railway Sciences. He is currently an associate researcher who worked in Signal and Communication Research Institute of China Academy of Railway Sciences. He has been engaged in the research and development of high speed railway communication system, comprehensive dispatching system, synthetic automation of marshalling yard, and comprehensive scientific management during past 12 years.

Lu Yan received the B.S degrees in Automation Science and Electronic Engineering from Beijing University of Aeronautics and Astronautics, Beijing, China, in 2005, the M.S degrees in Measuring and Testing Technologies and Instruments from Beijing University of Aeronautics and Astronautics in 2008, and in the PhD. Program of Traffic Information Engineering and Control in China Academy of Railway Sciences. She is currently an associate researcher of China Academy of Railway Sciences, and a manager in Science and Information Technology Department. Her research interests include railway signal and communication, integrated dispatching, highspeed railway earthquake early warning, disaster warning, etc. She has participated many national scientific projects in China and has rich experience in system.

Haixiang Liu received the B.S degrees in Traffic signal and control engineering from Shanghai Tiedao University, Shanghai, China, in 1996. He is currently a Professor with Signal and Communication Research Institute, China Academy of Railway Sciences. He has been engaged in the research and development of railway communication system, computer interlocking system, and railway traffic control system during past many years.

Ke Li received the Ph.D. degreee from Tianjin University, Tianjin, China, in 2011. He is currently an associate researcher at Signal and Communication Research Institute, China Academy of Railway Sciences Corporation Limited. His research interests include railway signal and communication, railway dynamic monitoring technology, and intelligent data fusion technology.

Jian Wang received the B.S. degree in Automation from Beijing Jiaotong University, Beijing, China, in 2005, the M.S. degree in Traffic Information Engineering and Control from Beijing Jiaotong University, Beijing, China, in 2008, and in the Ph.D program of Traffic Information Engineering and Control in China Academy of Railway Sciences. He is currently an Associate Researcher at Signal and Communication Research Institute, China Academy of Railway Sciences Corporation Limited, and an Information Technology Project Management Professional. His research interests include railway signal and communication, and railway intelligent control and dispatching.

\section{Funding}

This research was supported in part by the key Programs of Technological R\&D of China Academy of Railway Sciences under the Grant 1951TH6804.

\section{Declarations}

\section{Consent for publication}

Informed consent was obtained from all authors included in the study.

\section{Competing interests}

The authors declare that they have no competing interests.

\section{Received: 27 April 2021 Accepted: 17 July 2021}

Published online: 11 August 2021

\section{References}

1. C. Wang, A. Ghazal, B. Ai, Y. Liu, P. Fan, Channel measurements and models for high-speed train communication systems: a survey. IEEE Commun. Surv. Tutor. 18(2), 2016

2. B. Ai, Challenges toward wireless communications for high-speed railway. IEEE Trans. Intell. Transp. Syst. 15(5), 2143-2158 (2014)

3. T. Zhou, H. Li, Y. Wang, L. Liu, C. Tao, Channel modeling for future high-speed railway communication systems: a survey. IEEE Access. 52818-52826, 7 (2019)

4. Z. Jiang, C. Xu, J. Guan, Stochastic analysis of DASH-based video service in high-speed railway networks. IEEE Trans. Multimed. 21 (6), 1577-1592 (2019)

5. S. Xu, G. Zhu, B. Ai, A survey on high-speed railway communications: a radio resource management perspective. Comput. Commun. 12-28, 86 (2016)

6. Z. R. Wen, R. S. He, B. Ai, B. Zhang, M. Yang, W. Wang, Z. D. Zhong, H. X. Zhang, Measurement and modeling of LTE-railway channels in high-speed railway environment. Radio Sci. 55(4), 1-12 (2020)

7. R. Chen, W. Long, G. Mao, C. Li, Development trends of mobile communication systems for railways. IEEE Commun. Surv. Tutor. 20(4), 3131-3141 (2018)

8. S. Xu, G. Zhu, C. Shen, Y. Lei, in 2013 8th International Conference on Communications and Networking in China (CHINACOM), Delay-aware fair scheduling in relay-assisted high-speed railway networks (IEEE, 2013), pp. 17-21

9. C. Li, P. Liu, C. Zou, F. Sun, J. M. Cioffi, L. Yang, Spectral-efficient cellular communications with coexistent one- and two-hop transmissions. IEEE Trans. Veh. Technol. 65(8), 6765-6772 (2016)

10. C. Li, F. Sun, J. M. Cioffi, L. Yang, Energy efficient MIMO relay transmissions via joint power allocations. IEEE Trans. Circ. Syst. II Express Briefs. 6(7), 531-535 (2014)

11. J. Zhao, Y. Liu, Y. Gong, C. Wang, L. Fan, A dual-link soft handover scheme for C/U plane split network in high-speed railway. IEEE Access. 12473-12482, 6 (2018)

12. J. Zhao, Y. Liu, C. Wang, High-speed based adaptive beamforming handover scheme in LTE-R. IET Commun. 12(10), 1215-1222 (2018)

13. H. Yin, R. Jiang, Y. Xu, in 201810 th International Conference on Wireless Communications and Signal Processing (WCSP), An mmwave-based adaptive multi-beamforming scheme for high speed railway communications (IEEE, 2018), pp. 1-6 
14. W. Zhang, A beamforming scheme with doppler suppression for high-mobility wireless communications. IEEE Wirel. Commun. Lett. 9(10), 1768-1772 (2020)

15. C. Wang, R. T. Xu, Z. D. Zhong, in Proceedings of IEEE International Conference on Signal Processing, Distributed beamforming for high speed railway (IEEE, 2012), pp. 1410-1414

16. S. Xu, G. Zhu, C. Shen, Analysis and optimization of resource control in high-speed railway wireless networks. Math. Probl. Eng. 2014, 1-13 (2014)

17. S. Xu, G. Zhu, C. Shen, S. Li, Z. Zhong, in 2016 IEEE 83rd Vehicular Technology Conference (VTC Spring), Delay-aware dynamic resource management for high-speed railway wireless communications (IEEE, 2016), pp. 1-5

18. Z. Liu, J. Wu, P. Fan, in 2014 IEEE 79th Vehicular Technology Conference (VTC Spring), On the uplink capacity of high speed railway communications with massive mimo systems (IEEE, 2014), pp. 1-5

19. J. Yang, B. Ai, An effificient mimo channel model for LTE-R network in high-speed train environment. IEEE Trans. Veh. Technol. 68(4), 3189-3200 (2019)

20. C. Li, H. J. Yang, F. Sun, J. M. Cioffi, L. Yang, Multiuser overhearing for cooperative two-way multiantenna relays. IEEE Trans. Veh. Technol. 65(5), 3796-3802 (2016)

21. P. V. Craven, S. Craven, in Proceedings of the 2005 ASME/IEEE Joint Rail Conference, Security of ATCS wireless railway communications (IEEE, 2005), pp. 227-238

22. C. Meng, G. Wang, X. Dai, Secure energy-efficient transmission for SWIPT intelligent connected vehicles with imperfect CSI. IEEE Access. 7, 49-154658 (2019)

23. C. Meng, G. Wang, B. Yan, Energy efficiency optimization for secure SWIPT system. IEICE Trans. Commun. E103.B(5), 582-590 (2020)

24. Y. Cui, X. Fang, in The Sixth International Workshop on Signal Design and Its Applications in Communications, A physical layer secure wireless communication scheme for high speed railway (IEEE, 2013), pp. 114-117

25. Q. Ding, T. Xi, Lian Y., Joint power allocation scheme for distributed secure spatial modulation in high-speed railway. IEEE Syst. J. https://doi.org/10.1109/JSYST.2020.3008441

26. X. Gao, H. Han, K. Yang, J. An, Energy efficiency optimization for D2D communications based on SCA and GP method. China Commun. 14(3), 66-74 (2017)

27. Y. Zhou, C. Pan, P. L. Yeoh, Secure communications for UAV-enabled mobile edge computing systems. IEEE Trans. Commun. 68(1), 376-388 (2020)

28. H.-M. Wang, X. Zhang, UAV secure downlink NOMA transmissions: a secure users oriented perspective. IEEE Trans. Commun. 68(9), 5732-5746 (2020)

\section{Publisher's Note}

Springer Nature remains neutral with regard to jurisdictional claims in published maps and institutional affiliations.

\section{Submit your manuscript to a SpringerOpen ${ }^{\odot}$ journal and benefit from:}

- Convenient online submission

- Rigorous peer review

- Open access: articles freely available online

- High visibility within the field

- Retaining the copyright to your article

Submit your next manuscript at $\gg$ springeropen.com 